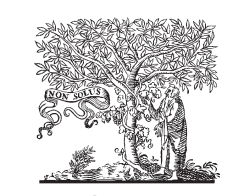

ELSEVIER

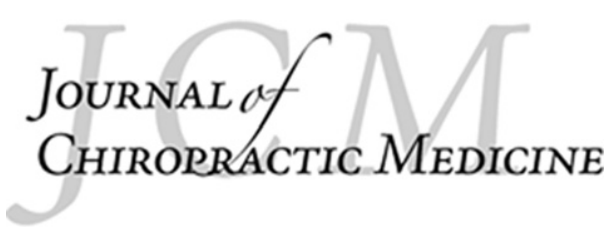

www.journalchiromed.com

\title{
Distal Embolic Brain Infarction Due to Recanalization of Asymptomatic Vertebral Artery Occlusion Resulting From Cervical Spine Injury: A Case Report
}

\section{Yaoki Nakao MD*, Hiroshi Terai MD}

\author{
Attending Physician, Daiyukai General Hospital, Ichinomiya, Japan
}

Received 12 November 2013; received in revised form 19 August 2014; accepted 23 August 2014

\section{Key indexing terms:}

Spinal cord injuries;

Cervical vertebrae;

Vertebral artery

\begin{abstract}
Objective: The purpose of this case report is to describe a patient with brain infarction due to recanalization of an occluded vertebral artery (VA) following closed reduction and open fixation of cervical spinal dislocation and to discuss the management of asymptomatic VA injuries associated with spine trauma.

Clinical Features: A 41-year-old Asian man experienced a C4-5 distractive-flexion injury manifesting with quadriplegia and anesthesia below the $\mathrm{C} 3$ cord level (including phrenic nerve paralysis), and bowel and bladder dysfunction. Magnetic resonance angiography and computed tomography angiography showed left extracranial VA(V2) occlusion and a patent contralateral VA. Intervention and Outcome: The patient was observed without antiplatelet and/or anticoagulation therapy and underwent open reduction and internal fusion of $\mathrm{C} 4 / 5$ and tracheostomy 8 hours after the injury. After surgery, supraspinal symptoms such as left horizontal nystagmus and left homonymous hemianopsia led to cranial computed tomography and magnetic resonance imaging, which showed left-side cerebellar infarction in the posterior inferior cerebellar artery territory and right-side posterior cerebral artery infarction. Magnetic resonance angiography and computed tomography angiography demonstrated patent bilateral VA (but hypoplastic right VA) and occluded right posterior cerebral artery. The injured VA was treated conservatively, and there were no other ischemic complications. Conclusion: The management of asymptomatic VA injury is controversial, with several treatment options available, including observation alone, antiplatelet therapy, anticoagulation therapy, or invasive intervention. Although there are some reports described where management with observation alone seems safe, serious attention should be given to the VA injury caused by cervical spine trauma.
\end{abstract}

(C) 2014 National University of Health Sciences.

\footnotetext{
* Corresponding author. 4918551. Tel.: +81 586721211; fax: +81 792728550 .

E-mail address: Nakao-ygc@umin.org(Y. Nakao).
} 


\section{Introduction}

Various nonpenetrating injuries, as well as penetrating injuries, have been associated with cerebrovascular injuries, not excepting cervical spine fracture and dislocation. Compared with carotid arterial injuries, vertebral artery (VA) injuries associated with blunt cervical spine trauma were thought to be infrequent because most of them were asymptomatic and because imaging studies were not performed routinely. Recent improvements in the imaging technology and increased use of screening protocols have led to a greater number of these injuries being identified. ${ }^{1}$ Traumatic VA injury can have disastrous consequences of basilar territory infarction and death. ${ }^{2}$ Although it is often assumed that reduction of a fracture without treatment of an associated asymptomatic VA injury is safe, ${ }^{3}$ when vertebrobasilar ischemia occurs, the mortality rate is $75 \%$ to $86 \% .{ }^{4}$ Vertebral artery injuries are relatively frequent and may be associated with significant morbidity and mortality in patients with cervical spine fractures or dislocations. ${ }^{2}$ Foremost in dealing with such an unusual but potentially devastating injury is determining whether the injury can be detected and effectively treated before complications occur.

Despite such a situation, well-defined treatment recommendations are still lacking. ${ }^{1}$

The frequency of vertebrobasilar ischemia in patients with cervical spine trauma is reported as low in many published papers, but there have been some case reports describing cervical spine injury associated with blunt VA injury. Many aspects of the management of VA injuries remain controversial, including the screening criteria, the diagnostic modality, and the optimal treatment for various lesions.

To contribute to further discussion of this topic, we present a case of brain infarction due to recanalization of the occluded VA following closed reduction and open fixation of cervical spinal dislocation, and discuss the management of asymptomatic VA injuries associated with spine trauma.

\section{Case Report}

A 41-year-old right-handed Asian man was attacked from behind by a cow and fell down. He received hyperflexion injury when he was struck on the top of the head. He did not lose consciousness but described immediate loss of power and sensation in both his arms and legs. He received emergency transport, was immobilized, and was transferred to the local hospital, where cervical spine trauma was suspected. There were no findings suggesting brain infarction in cranial computed tomography (CT). Unavailability of spine specialists prompted the patient's transfer to our institution, where he arrived 6 hours after injury.

Clinical assessment in the hospital found a Glasgow Coma Scale of 15 of 15 but complete neurologic deficits below the level of $\mathrm{C} 5$. Plain film radiographs obtained at this time showed a $\mathrm{C} 4$ on $\mathrm{C} 5$ dislocation (Fig 1). Further information regarding the severity of the injury was required, and so a cervical CT scan and magnetic resonance imaging were performed.

Computed tomographic scan showed a bifacet dislocation with more than $50 \%$ displacement of $\mathrm{C} 4$ on $\mathrm{C} 5$, and the magnetic resonance imaging revealed severely compressed dural tube at C4-C5 level and high signal intensity in the spinal cord on $\mathrm{T} 2$-weighted imaging (Fig 2). A sagittal CT showed bilateral dislocation of facet joints. Magnetic resonance angiography (MRA) showed complete occlusion of the right VA.

The patient was taken to the operating room 100 minutes after arrival. We performed closed reduction and posterior open surgery under general anesthesia. Pedicle screws on the left side and spinous process wiring were used for fixation, and local bone was grafted around C4-C5 facet joints bilaterally. Plain film radiographs obtained just after the surgery showed good fixation (Fig 3).

The patient demonstrated consciousness deterioration associated with repeated vomiting and left hemianopsia. These symptoms led to brain examination and studies. There were low dense lesions in the right posterior lobe and left cerebellum (Fig 4). Magnetic resonance angiography showed occlusion of right posterior cerebral artery (P2) and recanalization of occluded left VA (Fig 5). This seemed to be due to distal embolization of the clot around the occluded vessel. We did not administrate tissue plasminogen activator because the time of onset was unknown and because of the recent cervical spine surgery. Left hemianopsia had remained, although there was no change in his cervical spinal cord dysfunction.

Informed consent was obtained from the patient prior to publication of this case report and accompanying images. The document was completed by his wife because the patient could not sign it himself because of severe tetraplegia. The protocols for human procedures used in this study were approved by the ethics committee of our institution. 

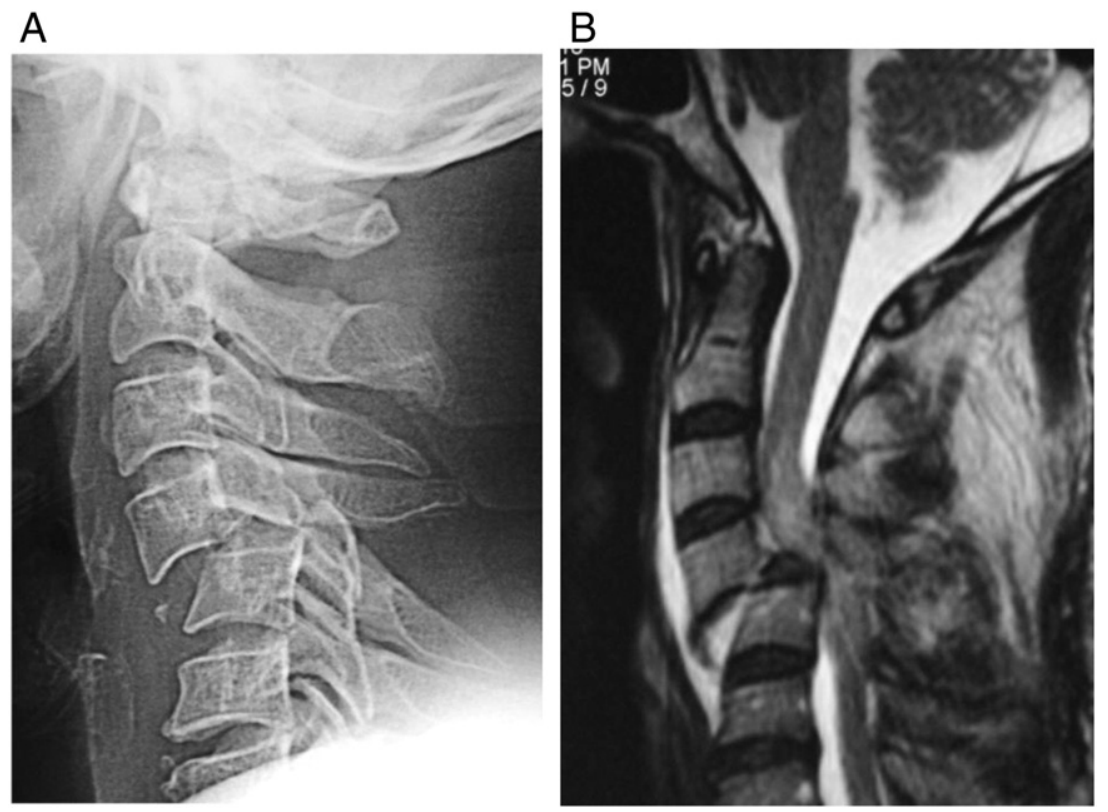

Fig 1. A, Plain lateral cervical spine radiograph shows the C4-5 dislocation. B, T2 fast spin echo sequence sagittal magnetic resonance image shows cord changes at the level of the C4-5 and the associated soft tissue damage.

\section{Discussion}

We present the case of a bilateral cervical facet joint dislocation associated with unilateral VA injury found in a 41-year-old man, treated by closed reduction and posterior fixation. After surgery, he demonstrated an episode of brain infarction that seemed to be caused by artery-toartery embolization originating from recanalized VA. Perhaps, VA occlusion on the dominant side caused by cervical spinal dislocation led to cerebellar infarction in the posterior inferior cerebellar artery territory due to hemodynamic compromise or arterial dissection.

The incidence of VA injury ranges from $0.075 \%$ to $0.77 \%$ of blunt trauma admission. ${ }^{1,4,5}$ When asymptom- atic patients are screened for blunt cerebrovascular injuries, the incidence rate rises to $1 \%$ of all blunt trauma patients and as high as $2.7 \%$ in patients with an Injury Severity Score greater than 16. 6,7 The possibility of association with cerebrovascular injury rises up to $17.2 \%$ to $25.5 \%$ in "high-risk" patients who satisfy the Denver screening criteria for blunt cerebrovascular injury. 8,9

Some reports ${ }^{10}$ have described patients with cervical spinal trauma who had rapidly fatal neurologic disorders suggestive of serious vertebrobasilar insufficiency. Indeed, the time course of vertebrobasilar ischemia secondary to VA occlusion is variable, ranging from a few hours to several years. In our case, the time course was 24 hours after the injury and 12 hours after the reductive surgery, respectively.
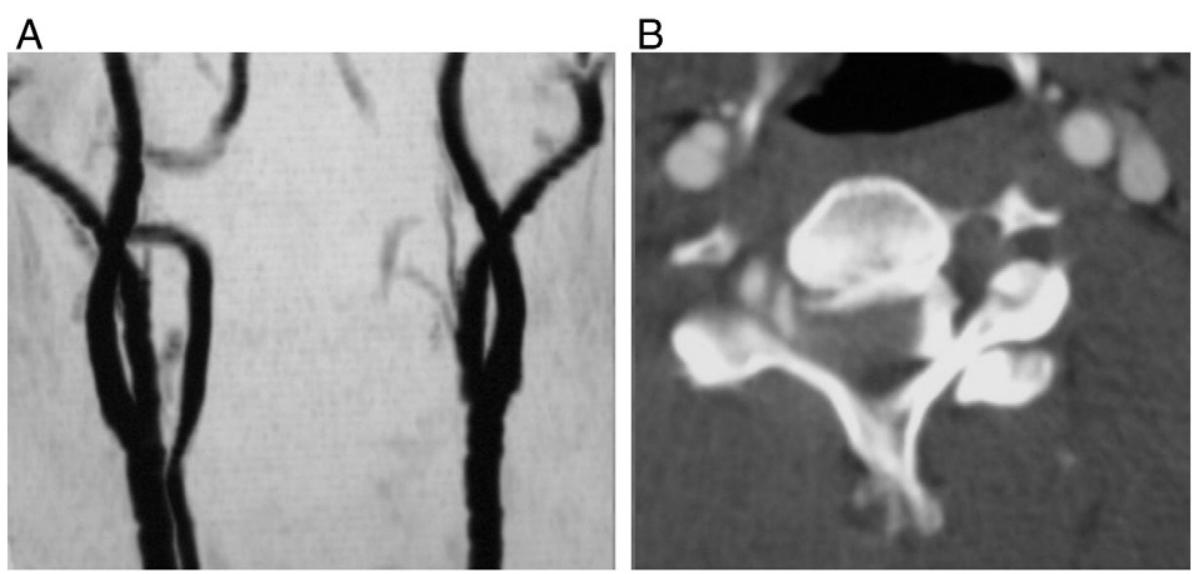

Fig 2. A, MRA shows occluded left VA. B, Axial CTA image shows complete occlusion of the left VA at the level of the dislocation. 
A

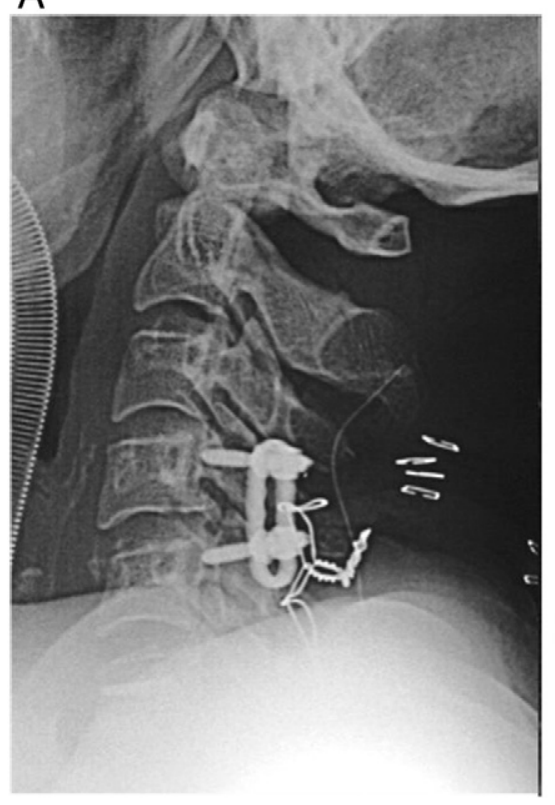

B

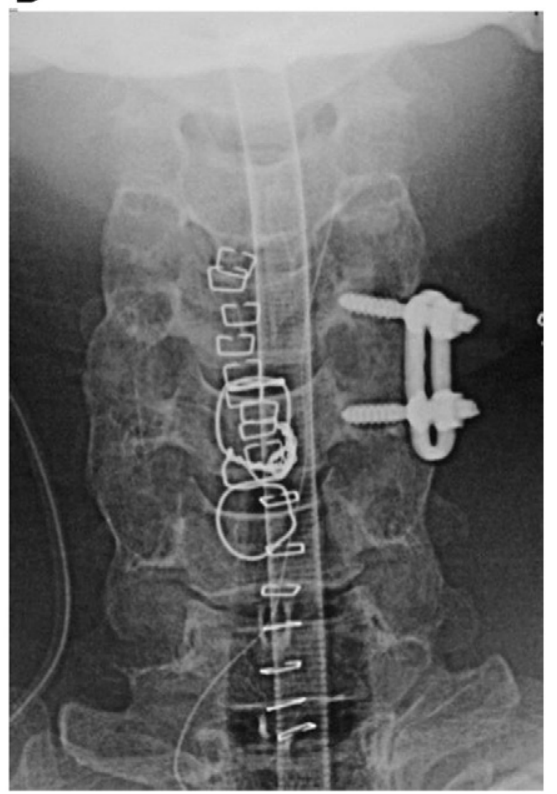

Fig 3. A and B, Plain radiographs taken after surgery shows solid fixation of C4-5.

Vessel occlusion secondary to damage to the intimal lining may be acute or delayed and involve a progression of platelet aggregation, followed by thrombus formation and then thromboembolic occlusion. ${ }^{3,11}$ Direct injury from the surrounding structure such as bony tip also may result in VA injury. External layer injury may occur and cause pseudoaneurysm and vessel tear in patients exposed to greater force. ${ }^{12} \mathrm{In}$ most cases, the V2 segment that runs through the vertebral foramen of C6 until C2 is affected ${ }^{8,12,13}$; and the vascular lesion usually presents next to the cervical fractures because of its fixation to the spine within the transverse foramen from $\mathrm{C} 1$ through $\mathrm{C} 6 .^{3}$
The type of fracture associated with VA injury is variable. Two types of cervical spine injuries are especially associated with a higher risk for VA injury: (1) fractures involving the transverse foramen and (2) subluxations. These types constitute $46 \%$ to $75 \%$ of traumatic VA injuries associated with cervical trauma. ${ }^{14,15}$

A high clinical index of suspicion remains the most important factor in making the diagnosis. ${ }^{16}$ Clinicians should consider the dangers of VA injury in all patients who have sustained any type of cervical spine injury. Once a patient is found to be at risk of VA injury, imaging studies should be taken to rule out VA injury and avoid neurological deterioration, even if there are
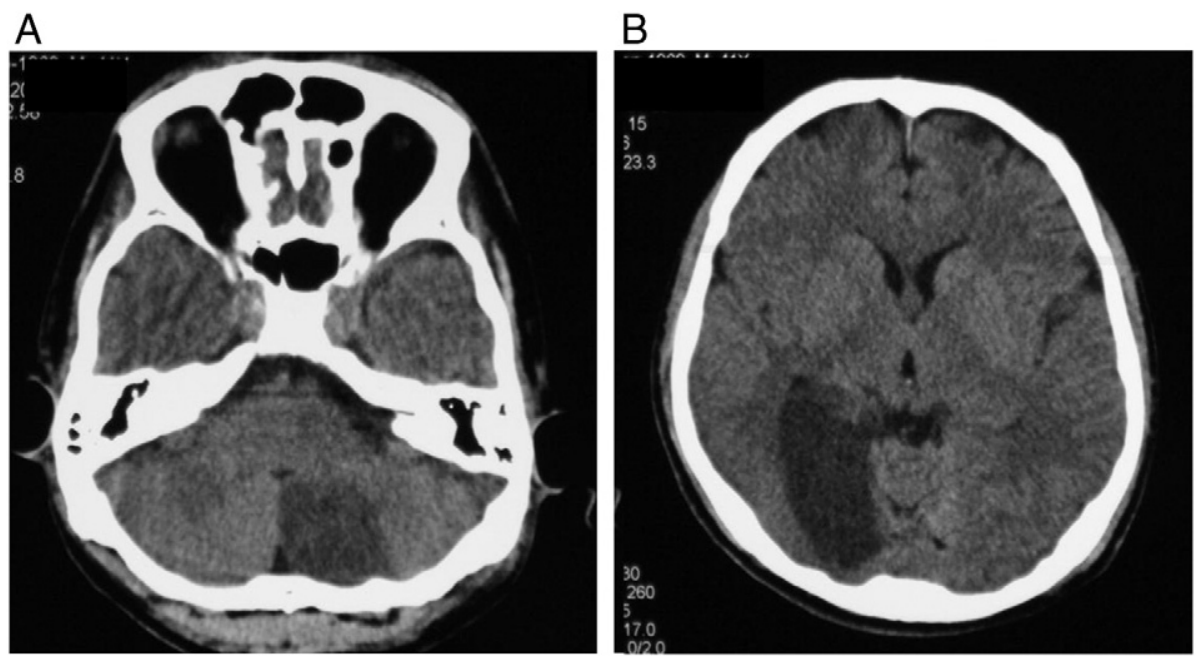

Fig 4. Brain CT scan shows brain infarction in left posterior inferior cerebellar artery (A) and right posterior cerebral artery territory (B). 

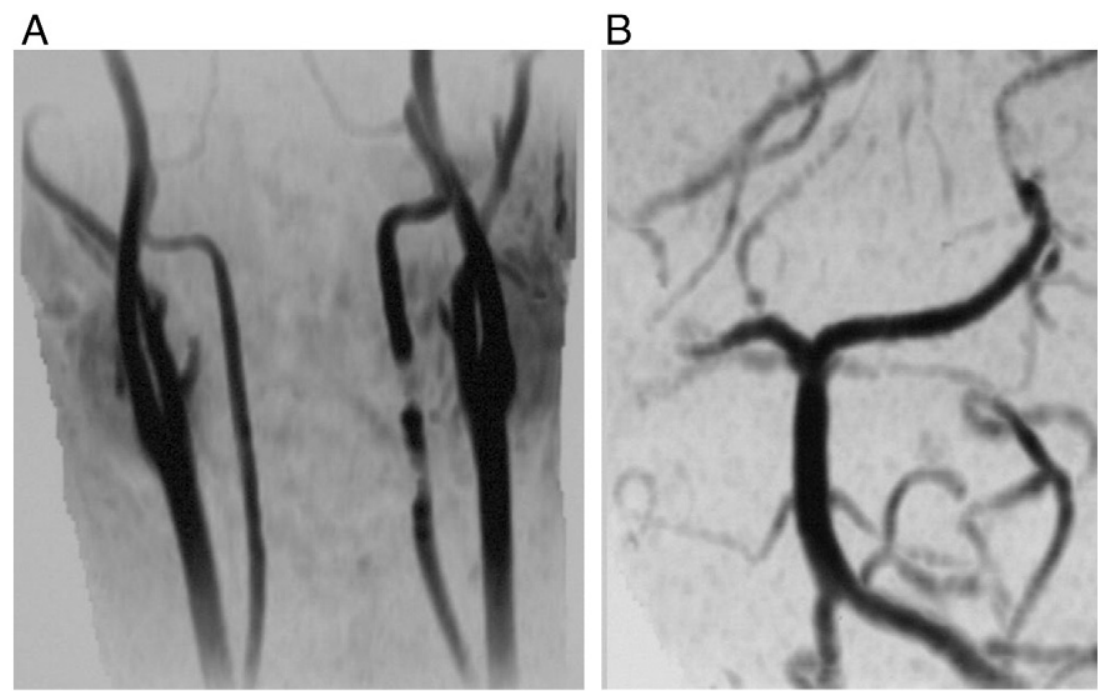

Fig 5. MRA demonstrates recanalized left VA and occluded right P2.

no symptoms of vertebrobasilar insufficiency such as altered consciousness, dysarthria, blurred vision, nystagmus, ataxia, or dysphagia. ${ }^{17}$

Although there have been no definite standards or guidelines as to what is the most important and optimal imaging study for patients with suspected VA injury, a broad-scale, less invasive, low-cost, and fast screening program is probably the best way to diagnose these lesions. ${ }^{7}$ Magnetic resonance angiography is quite accurate in the detection of near or total occlusion of the V2 segment noninvasively. ${ }^{12,14,17,18}$ On the other hand, slow blood flow in the small vessels on MRA can be confused with occlusion as a result of insufficient resolution. ${ }^{19}$ Conventional angiography is superior to MRA in the detection of nonoccluded intimal disruption, which occasionally causes distal embolization. However, it has not been performed as the first-line diagnostic procedure in recent years because of its invasiveness. Computed tomography angiography (CTA) with more than 16-slice scanners has been shown to have sensitivity and specificity close to those of conventional angiography. ${ }^{18,20}$. Additionally, it is more widely available, has fewer contraindications, and provides greater spatial resolution than MRA. Computed tomography angiography may therefore become an acceptable alternative of conventional angiography. We willingly indicate multidetector CTA in patients with "high-risk" cervical spine fractures.

Treatment of blunt VA injury remains controversial. Reported treatments for asymptomatic VA injury include supportive management, surgical ligation, radiologic embolization, systemic heparinization, and antiplatelet therapy. Management strategies proposed are based on the radiological grade and clinical severity of cervical spine trauma and VA injury, but are still not standardized and vary from anticoagulation to ligation and thrombectomy.

The most frequently used specific treatment is anticoagulation. There have been a number of studies attempting to evaluate the impact of antithrombotic agents on the progression or development of sequelae of blunt cerebrovascular injuries. A series of retrospective studies ${ }^{12,14,21}$ found that administration of antithrombotic agents reduces the rate of neurologic sequelae after blunt cerebrovascular injuries. The rationale for administration of anticoagulant and/or antiplatelet has been to (1) minimize clot formation at the site of intimal injury; (2) decrease further propagation of clot which has formed, allowing the internal fibrinolytic systemic to dissolve the clot; and 3) prevent embolization. However, systemic administration introduces risks of hemorrhagic complications, especially in multisystem trauma victims. We can reduce the incidence of bleeding complications by avoiding bolus heparin dosing and targeting a lower partial thromboplastin time. ${ }^{4}$ Although there has not been a direct, controlled comparison of heparinization vs antiplatelet agents in the prevention of cerebral vascular event after VA injury, a number of studies performed subgroup analysis in an attempt to address this question.

There has been discussion of preemptive embolization of blunt VA injury as primary therapy. This is based on the premise that occlusion of unilateral VA is generally well tolerated. The presence of VA agenesis has been found to be $1.8 \%$ to $3.1 \%$ in autopsy study, ${ }^{22}$ 
and unilateral VA dominance is uncommon, Hoshino et $\mathrm{a}^{23}$ performed unilateral VA ligation on 15 patients without adverse sequelae on long-term follow-up. However, routine embolization introduces the risk that the contralateral VA may acquire transient flow disturbances, resulting in infarction. There have also been several preliminary studies that have indicated the safety and feasibility of catheter-directed therapy to include embolization of pseudoaneurysms and stenting of intimal injuries. ${ }^{24}$

We gave priority in this case to cervical fixation over treatment of the asymptomatic VA injury, although we understood the risk of distal embolization. That was because we tried to decompress the neural structure and stabilize the cervical spine promptly to prevent neurological deterioration and to initiate early rehabilitation. We also wanted to avoid bleeding complication in case tracheostomy was required following the cervical fixation. Another approach to achieve reduction and fixation could have been to perform proximal occlusion interventionally. Indeed, cervical spine injured patients with associated VA injury should ideally be in conjunction with an interventionist who has the ability to accomplish interventional coil occlusion.

\section{Conclusion}

The management of asymptomatic VA injury is controversial, with several treatment options available. Although some reports have indicated that management with observation alone seems safe, serious attention should be paid to a potential ischemic event due to VA injury caused by cervical spine trauma. For patients with cervical spine injuries who need immediate fixation, the help of an interventionist may be advised $^{25-29}$.

\section{Funding Sources and Conflicts of Interest}

No funding sources or conflicts of interest were reported for this study.

\section{References}

1. Berne JD, Norwood SH, McAUley CE, et al. Helical computed tomographic angiography: an excellent screening test for blunt cerebrovascular injury. J Trauma 2004;57:11-9.
2. Yoshihara H, VanderHeiden TF, Harasaki Y, et al. Fatal outcome after brain stem infarction related to bilateral artery occlusion -case report of a detrimental complication of cervical spine trauma. Patient Saf Surg 2011;5:18.

3. Willis BK, Greiner F, Orrison WW, et al. The incidence of vertebral artery injury after midcervical spine fracture or subluxation. Neurosurgery 1994;34:435-42.

4. Biffl WL, Moore EE, Eliott JP, et al. The devastating potential of blunt vertebral arterial injuries. Ann Surg 2000;231:672-81.

5. Bula WI, Loes DJ. Trauma to the cerebrovascular system. Neuroimaging Clin North Am 1994;4:753-772.

6. Biffl WL, Moore EE, Ryu RK, et al. The unrecognized epidemic of blunt carotid arterial injuries: early diagnosis improves neurologic outcome. Ann Surg 1998;228:462-70.

7. Mutze S, Rademacher G, Matthes G, et al. Blunt cerebrovascular injury in patients with blunt multiple trauma: diagnostic accuracy of duplex Doppler US and early CT angiography. Radiology 2005;237:884-92.

8. Biffl WL, Moore EE, Offner PJ, et al. Optimizing screening for blunt cerebrovascular injuries. Am J Surg 1999;178:517-22.

9. Louw JA, Mafoyane NA, Small B, et al. Occlusion of the vertebral artery in cervical spine dislocations. J Bone Joint Surg (Br) 1990;72:679-81.

10. Okuyama T, Minamida Y, Sasaki T, Horikawa D, Izumiyama O, Mizuguchi M. Traumatic occlusion of the bilateral vertebral arteries associated with fracture of the cervical spine: a case report. Neurol Surg 1987;15:1141-5.

11. Taneichi H, Suda K, Kajino T, et al. Traumatically induced vertebral artery occlusion associated with cervical spine injuries: prospective study using magnetic resonance angiography. Spine 2005;30:1955-62.

12. Miller PR, Fabian TC, Bee TK, et al. Blunt cerebrovascular injuries: diagnosis and treatment. J Trauma 2001;51:279-86.

13. Vaccaro AR, Harkey HL, Touchstone DA, et al. Long-term evaluation of vertebral artery injuries following cervical spine trauma using magnetic resonance angiography. Spine 1998;23:789-94.

14. Cothren CC, Moore EE, Biffl WL, et al. Cervical spine fracture patterns predictive of blunt vertebral injury. J Trauma 2003;55:811-3.

15. Parent AD, Harkey HL, Touchstone DA, et al. Lateral cervical spine dislocation and vertebral artery injury. Neurosurgery 1992;31:501-9.

16. Bok AP, Peter JC. Carotid and vertebral artery occlusion after blunt cervical injury: the role of MR angiography in early diagnosis. J Trauma 1996;40:968-72.

17. Deen Jr HG, McGirr SJ, et al. Vertebral artery injury associated with cervical spine fracture. Spine 1992;17:230-4.

18. Biffl WL, Ray Jr CE, Moore EE, et al. Treatment-related outcomes from blunt cerebrovascular injuries: importance of routine follow-up angiography. Ann Surg 2002;235:699-707.

19. Miller PR, Fabian TC, Croce MA, et al. Prospective screening for blunt cerebrovascular injuries: analysis of diagnostic modalities and outcomes. Ann Surg 2002;236:386-95.

20. Bromberg WJ, Collier BC, Diebel LN, et al. Blunt cerebrovascular injury practice management guidelines: the eastern association for the surgery of trauma. J Trauma 2010;68:471-7.

21. Cothren CC, Moore EE, Biffl WL, et al. Anticoagulation is the gold standard therapy for blunt carotid injuries to reduce stroke rate. Arch Surg 2004;139:609-13. 
22. Saito T, Kamisawa O, Kaminishi Y, et al. Isolated traumatic vertebral pseudoaneurysm: report of a case. Surg Today 2003;33:145-7.

23. Hoshino Y, Kurokawa T, Nakamura K, et al. A report on the safety of unilateral vertebral artery ligation during cervical spine surgery. Spine 1996;21:1454-7.

24. Coldwell DM, Novak Z, Ryu RK, et al. Treatment of posttraumatic internal carotid arterial pseudoaneurysms with endovascular stents. J Trauma 2000;48:470-2.

25. Levy C, Laissy JP, Raveau V, et al. Carotid and vertebral artery dissections: three-dimensional time-of-flight MR angiography and MR imaging versus conventional angiography. Radiology 1994;190:326-9.
26. Parbhoo AH, Govender S, Corr P. Vertebral artery injury in cervical spine trauma. Injury 2001;32:565-8.

27. Sack JA, Etame AB, Shah GV, et al. Management and outcomes of patients undergoing surgery for traumatic cervical fracturesubluxation associated with an asymptomatic vertebral artery injury. J Spinal Disord Tech 2009;22:86-90.

28. Tudorache C, Moldovanu A, Esanu A, et al. The posttraumatic occlusion of the vertebral artery case presentation. Rom Neurosurg 2010;17:238-45.

29. Vertinsky AT, Schwartz NE, Fischbein NJ, et al. Comparison of multidetector CT angiography and MR imaging of cervical artery dissection. AJNR Am J Neuroradiol 2008;29:1753-60. 